\title{
Design of Minimax Broadband Beamformers that are Robust to Microphone Gain, Phase, and Position Errors
}

\author{
R. C. Nongpiur, Member, IEEE
}

\begin{abstract}
Broadband beamformers with small-size microphone arrays are known to be highly sensitive to microphone imperfections. A new method for the design of minimax broadband beamformers that are robust to microphone gain, phase, and position errors is proposed. In the method, the maximum variations in the microphone errors are used in formulating a convex optimization problem where the worst-case passband error is minimized under the constraint that the worst-case stopband error is below a prescribed level. To include the microphone imperfections in the optimization problem, we developed a suitable model that incorporates the variations due to the microphone errors and at the same time is efficient to compute. An important advantage of the proposed method is the availability of corresponding worstcase passband- and stopband-error bounds for the beamformer that has been designed; a second advantage is that it does not require the probability distributions of microphone errors. We then describe a two-phase method where the proposed method is used in the first phase to derive the passband and stopband error constraints for solving an optimization problem in the second phase where the white noise gain (WNG) of the beamformer is maximized. In our experiments, we compare beamformers designed using the proposed method, the two-phase method and a modified version of a competing method. Experimental results show that beamformers designed using the proposed method have much better performance than those of the modified competing method and comparable performance with those of the two-phase method; however, unlike the two-phase method, the proposed method provides the additional guarantee that the errors will always lie within the worst-case error bounds.
\end{abstract}

Index Terms-acoustic beamforming, broadband beamformer, constrained optimization, speech enhancement

\section{INTRODUCTION}

Microphone arrays are widely used in speech communication applications such as hands-free telephony, hearing aids, speech recognition, and teleconferencing systems. A technique that is widely used with microphone arrays to enhance a speech signal from a preferred spatial direction is beamforming [1]. In general, the beamforming approach can be fixed or adaptive, depending upon whether the spatial directivity pattern is fixed or varies adaptively on the basis of incoming data. Though adaptive beamforming performs better when the acoustic environment is time-varying, fixed beamforming are preferred in applications where the direction of the sound

Copyright (c) 2013 IEEE. Personal use of this material is permitted. However, permission to use this material for any other purposes must be obtained from the IEEE by sending an email to pubs-permissions@ieee.org.

R. C. Nongpiur is with the Department of Electrical and Computer Engineering, University of Victoria, Victoria, BC, Canada V8W 3P6 e-mail: rnongpiu@ece.uvic.ca

Manuscript submitted August 2013. source is fixed, such as in in-car communication systems [2] or in hearing aids. In addition, fixed beamformers also have lower computational complexity and are easier to implement.

In many beamformer applications, such as in-car communication systems, voice recognition systems, video conferencing systems, etc., there is often a need to ensure that the gain across the passband has little variation from unity while that in the stopband is always below a prescribed level. At the same time, a passband with good linear-phase characteristics is usually preferred to avoid any signal distortion. Consequently, for the design of such beamformers a straightforward approach is to formulate the optimization problem in terms of the minimax of the appropriate error functions.

In [3]-[8], designs of broadband beamformers that are not constrained by the size of the array aperture or are based on the assumption of ideal or known microphone characteristics have been proposed. However, in certain applications such as in hearing aid and in-car communication systems there are physical constraints on the array aperture size such that the wavelength of the signal in the lower end of the frequency band is much longer than the maximum allowed aperture length. Consequently, as evident from earlier designs for superdirective narrowband arrays [9]-[13], broadband beamformers designed for physically-compact applications can likewise become very sensitive to errors in array imperfections and therefore robustness constraints need to be incorporated in the design.

Several robust broadband beamformer designs have been proposed in recent years. In [14], robustness is achieved by bounding the norm of the error matrices while in [15]-[18], the white noise gain (WNG) is incorporated in the design; the use of the WNG constraint is not new and has been used in earlier beamformer designs to ensure robustness in superdirective beamformers [9]-[11]. Then in [20]-[23], the statistics of the microphone characteristics are taken into account to derive broadband beamformers that are robust to microphone mismatches while in [24] the worst-case mean performance is optimized. However, a drawback with the methods in [14]-[24] is that there is no straightforward way of knowing the worst-case performance of the beamformer, since they are based on optimizing the mean performance. In [25], a minimax approach that optimizes the worst-case performance of prescribed combinations of microphone gain and phase errors was proposed. The approach, however, becomes computationally very expensive as the number of combinations is increased, and is practically feasible only when gain errors are 
considered. In [19], a method for designing near-field broadband beamformers that optimizes the worst-case performance was proposed. However, the method reported in [19] assumed beamformers with either gain and phase errors or position errors, but not all three errors simultaneously.

In this paper, we seek to remove the drawback in existing methods by developing a robust beamformer design method that is based on optimizing the worst-case performance. In the method, the maximum variations in the microphone errors, namely, gain, phase and position errors, are used in the formulation of a convex optimization problem where the worstcase passband error is minimized under the constraint that the worst-case stopband error is below a prescribed level. To include the microphone imperfections in the optimization, we developed a suitable model that incorporates the variations due to the microphone errors and is at the same time efficient to compute. An important advantage of the proposed method is the availability of corresponding worst-case passband and stopband error bounds for the beamformer that has been designed; a second advantage is that it does not require the probability distribution of microphone errors. We then describe a two-phase method where the proposed method is used in the first phase to derive the passband and stopband error constraints for solving an optimization problem in the second phase where the WNG of the beamformer is maximized. In our experiments, we compare beamformers designed using the proposed method, the two-phase method and a modified version of the method in [19]. Experimental results show that beamformers designed using the proposed method have much better performance than those of the modified competing method and comparable performance with those of the twophase method; however, unlike the two-phase method, the proposed method provides the additional guarantee that the errors will always lie within the worst-case error bounds.

The paper is organized as follows. In Section II, we describe the filter-and-sum beamformer in far field and incorporate the microphone imperfections in the error formulations. Then in Section III, we use the error formulations from Section II to derive the optimization problem for the proposed design method. In addition, we also describe the two-phase method where the WNG is maximized. In Section IV, we describe an efficient method to compute the microphone-error-variation model while in Section V, performance comparisons between the proposed method, the two-phase method, and a modified competing method are carried out. Conclusions are drawn in Section VI.

\section{FAR-FIELD BROADBAND BEAMFORMING}

In this paper, we assume a far-field signal impinging on a linear microphone array that is realized as a filter-and-sum beamformer, as shown in Fig. 1. The microphones are assumed to be omnidirectional and the filters are FIR. If $N$ is the number of microphones and $L$ is the length of each filter, the response of the filter-and-sum beamformer is given by

$$
B(\omega, \theta)=\sum_{n=0}^{N-1} \sum_{l=0}^{L-1} x_{n, l} g_{n, l}(\omega, \theta)
$$

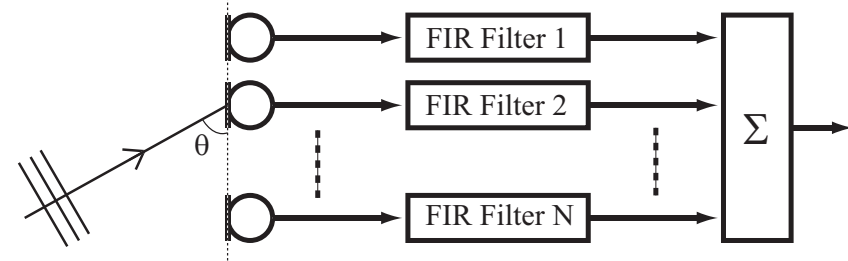

Fig. 1. Filter and sum broadband beamformer.

where

$$
g_{n, l}(\omega, \theta)=\exp \left[-j \omega\left(\frac{f_{s} d_{n} \cos \theta}{c}+l\right)\right]
$$

$\omega$ is the frequency in radians, $\theta$ is the direction of arrival, $c$ is the speed of sound in air, $f_{s}$ is the sampling frequency, $d_{n}$ is the distance of the $n$th microphone from the origin, and $x_{n, l}$ is the $l$ th coefficient of $n$th FIR filter. In matrix form, (1) can be expressed as

$$
B(\mathbf{x}, \omega, \theta)=\sum_{n=0}^{N-1} \mathbf{g}_{n}(\omega, \theta)^{T} \mathbf{x}_{n}=\mathbf{g}(\omega, \theta)^{T} \mathbf{x}
$$

where

$$
\begin{aligned}
\mathbf{x}^{T} & =\left[\begin{array}{lll}
\mathbf{x}_{0}^{T} & \mathbf{x}_{1}^{T} \cdots & \mathbf{x}_{N-1}^{T}
\end{array}\right] \\
\mathbf{g}(\omega, \theta)^{T} & =\left[\begin{array}{lll}
\mathbf{g}_{0}(\omega, \theta)^{T} & \mathbf{g}_{1}(\omega, \theta)^{T} \cdots & \mathbf{g}_{N-1}(\omega, \theta)^{T}
\end{array}\right](5) \\
\mathbf{x}_{n} & =\left[\begin{array}{lll}
x_{n, 0} & x_{n, 1} \cdots & x_{n, L-1}
\end{array}\right]^{T} \\
\mathbf{g}_{n}(\omega, \theta) & =\left[\begin{array}{lll}
g_{n, 0}(\omega, \theta) & g_{n, 1}(\omega, \theta) \cdots & g_{n, L-1}(\omega, \theta)
\end{array}\right]^{T}(7)
\end{aligned}
$$

If $\theta_{d}$ is the desired steering angle of the beamformer, the WNG of the beamformer is given by [1]

$$
G_{w}(\mathbf{x}, \omega)=\frac{\left|B\left(\mathbf{x}, \omega, \theta_{d}\right)\right|^{2}}{\sum_{n=0}^{N-1}\left|\sum_{l=0}^{L-1} x_{n, l} e^{-j \omega l}\right|^{2}}=\frac{\left|\mathbf{g}\left(\omega, \theta_{d}\right)^{T} \mathbf{x}\right|^{2}}{\|\mathbf{A}(\omega) \mathbf{x}\|_{2}^{2}}
$$

where

$$
\begin{aligned}
\mathbf{A}(\omega) & =\mathbf{I}_{N} \otimes \mathbf{a}(\omega)^{T} \\
\mathbf{a}(\omega)^{T} & =\left[1 e^{-j \omega} \cdots e^{-j(L-1) \omega}\right]^{T}
\end{aligned}
$$

$\mathbf{I}_{N}$ is an $N \times N$ identity matrix, $\otimes$ is the Kronecker product, and $\|\mathbf{v}\|_{2}$ is the $L_{2}$ norm of vector $\mathbf{v}$.

\section{A. Beamformer Response With Microphone Errors}

Let us consider a beamformer where the microphones have gain, phase, and position errors. If $k_{n}, \eta_{n}$, and $\hat{d}_{n}$ are the mean values of the gain, phase, and position of the $n$th microphone and $\Delta k_{n}, \Delta \eta_{n}$, and $\Delta \hat{d}_{n}$ their corresponding deviations, the response of the beamformer can be expressed as

$$
\hat{B}(\mathbf{x}, \omega, \theta)=\sum_{n=0}^{N-1} \kappa_{n}(\omega, \theta) \hat{\mathbf{g}}_{n}(\omega, \theta)^{T} \mathbf{x}_{n}
$$

where

$$
\kappa_{n}(\omega, \theta)=\left(k_{n} \pm \Delta k_{n}\right) e^{j\left(\eta_{n} \pm \Delta \eta_{n} \pm \frac{\omega \Delta \hat{d}_{n} \cos \theta}{c}\right)}
$$




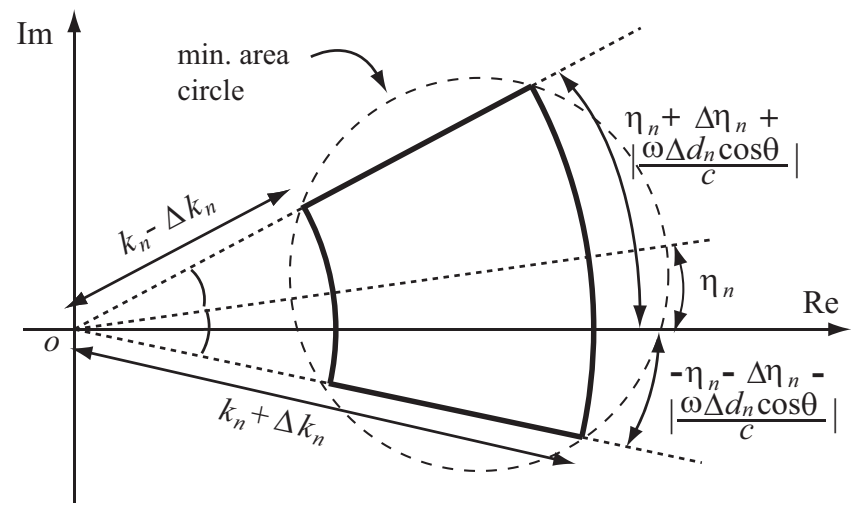

Fig. 2. Plot of the variations of $\kappa_{n}$ due to errors in gain, phase, and position of the $n$th microphone.

and $\hat{\mathbf{g}}_{n}(\omega, \theta)$ is the corresponding value of $\mathbf{g}_{n}(\omega, \theta)$ when $\hat{d}_{n}$ is used in place of $d_{n}$ in (2). From (12), it is apparent that $\kappa_{n}$ will vary with $\omega$ and $\theta$ only if there is position error. In the complex plane, the variation of $\kappa_{n}(\omega, \theta)$ will correspond to a sector of an annulus as shown, for example, in Fig. 2. To make the variations in $\kappa_{n}(\omega, \theta)$ amenable for optimization we approximate the variations by a circular model. More specifically, we find the minimum area circle that encloses $\kappa_{n}(\omega, \theta)$ in the complex plane, as denoted by the dashed lines in Fig. 2. A representation of $\kappa_{n}(\omega, \theta)$ using such as circle is given by

$$
\hat{\kappa}_{n}(\omega, \theta)=\mathbf{u}^{T} \mathcal{C}_{n}(\omega, \theta)
$$

where

$$
\begin{aligned}
\mathbf{u} & =\left[\begin{array}{ll}
1 & j
\end{array}\right]^{T} \\
\mathcal{C}_{n}(\omega, \theta) & =\left\{\mathbf{q}_{n}(\omega, \theta)+\mathbf{P}_{n}(\omega, \theta) \mathbf{v}_{n} \mid\left\|\mathbf{v}_{n}\right\|_{2} \leq 1\right\}
\end{aligned}
$$

parameter $\mathbf{q}_{n}(\omega, \theta) \in \mathbf{R}^{2 \times 1}$ corresponds to the center of the circle in the complex plane and matrix $\mathbf{P}_{n} \in \mathbf{R}^{2 \times 2}$ describes the variation of the errors within a circle of radius $r_{\chi}$, given by

$$
r_{\chi}=\left\|\mathbf{P}(\omega, \theta)^{T} \mathbf{u}\right\|_{\infty}
$$

An efficient method for computing the parameters of the circular model, $\mathcal{C}_{n}(\omega, \theta)$, is described in Section IV. Substituting (13) in (11) we get the beamformer response that is based on the circular model as

$$
\begin{aligned}
\hat{B}_{c}(\mathbf{x}, \omega, \theta)= & \sum_{n=0}^{N-1} \mathbf{u}^{T}\left[\mathbf{q}_{n}(\omega, \theta)+\right. \\
& \left.\mathbf{P}_{n}(\omega, \theta) \mathbf{v}_{n}\right] \hat{\mathbf{g}}_{n}(\omega, \theta)^{T} \mathbf{x}_{n}
\end{aligned}
$$

Assuming that that the microphone error characteristics are the same for each element of the array, we can set $\mathbf{P}(\omega, \theta)=$ $\mathbf{P}_{1}(\omega, \theta)=\ldots=\mathbf{P}_{N}(\omega, \theta)$ and $\mathbf{q}(\omega, \theta)=\mathbf{q}_{1}(\omega, \theta)=\ldots=$ $\mathbf{q}_{N}(\omega, \theta)$. Taking the absolute value of $\hat{B}_{c}(\mathbf{x}, \omega, \theta)$ we get the inequality

$$
\begin{array}{r}
\left|\hat{B}_{c}(\mathbf{x}, \omega, \theta)\right| \leq\left|\mathbf{u}^{T} \mathbf{q}(\omega, \theta) \sum_{n=0}^{N-1} \hat{\mathbf{g}}_{n}(\omega, \theta)^{T} \mathbf{x}_{n}\right|+ \\
\sum_{n=0}^{N-1}\left|\mathbf{u}^{T} \mathbf{P}(\omega, \theta) \mathbf{v}_{n} \hat{\mathbf{g}}_{n}(\omega, \theta)^{T} \mathbf{x}_{n}\right|
\end{array}
$$

As shown in Appendix A of [26], the worst-case magnitude of the beamformer response can be expressed as

$$
\begin{aligned}
\sup _{\mathbf{v}_{1}, \ldots, \mathbf{v}_{n}}\left|\hat{B}_{c}(\mathbf{x}, \omega, \theta)\right|=\mid & \mathbf{u}^{T} \mathbf{q}(\omega, \theta) \sum_{n=0}^{N-1} \hat{\mathbf{g}}_{n}(\omega, \theta)^{T} \mathbf{x}_{n} \mid+ \\
& \left\|\mathbf{P}(\omega, \theta)^{T} \mathbf{u}\right\|_{\infty} \sum_{n=0}^{N-1}\left|\hat{\mathbf{g}}_{n}(\omega, \theta)^{T} \mathbf{x}_{n}\right|
\end{aligned}
$$

In a similar manner, if $B_{d}(\omega, \theta)$ is the desired response of the beamformer, the worst-case error is given by

$$
\begin{aligned}
& \sup _{\mathbf{v}_{1}, \ldots \hat{B}_{n}}\left|\hat{B}_{c}(\mathbf{x}, \omega, \theta)-B_{d}(\omega, \theta)\right|= \\
& \left|\mathbf{u}^{T} \mathbf{q}(\omega, \theta) \sum_{n=0}^{N-1} \hat{\mathbf{g}}_{n}(\omega, \theta)^{T} \mathbf{x}_{n}-B_{d}(\omega, \theta)\right|+ \\
& \left\|\mathbf{P}(\omega, \theta)^{T} \mathbf{u}\right\|_{\infty} \sum_{n=0}^{N-1}\left|\hat{\mathbf{g}}_{n}(\omega, \theta)^{T} \mathbf{x}_{n}\right|
\end{aligned}
$$

\section{The OPtimizATION PROBLEM}

In this section, we use the worst-case error expressions derived in Section II-A to formulate a convex optimization problem for designing broadband beamformers that are robust to the sensor imperfections. We also describe a two-phase design method that maximizes the WNG in order to compare with the proposed design method.

\section{A. Proposed Design Method}

If $\Omega$ is defined as the frequency band, and $\Theta_{p}$ and $\Theta_{s}$ as the passband and stopband angular regions, respectively, the optimization problem can be formulated by minimizing the worst-case passband error under the constraint that the worstcase stopband error is above a prescribed level; that is

$$
\begin{array}{cl}
\operatorname{minimize} & \max _{\omega_{i}, \theta_{p}}\left\{\sup _{\mathbf{v}_{1}, \ldots, \mathbf{v}_{n}}\left|\hat{B}\left(\mathbf{x}, \omega_{i}, \theta_{p}\right)-B_{d}\left(\omega_{i}, \theta_{p}\right)\right|\right\} \\
\text { subject to: } & \max _{\omega_{i}, \theta_{s}}\left\{\sup _{\mathbf{v}_{1}, \ldots, \mathbf{v}_{n}}\left|\hat{B}\left(\mathbf{x}, \omega_{i}, \theta_{s}\right)\right|\right\} \leq \Gamma_{s b}^{(u b)}
\end{array}
$$

where $\theta_{p} \in \Theta_{p}, \theta_{s} \in \Theta_{s}$, and $\omega_{i} \in \Omega$ and $\Gamma_{s b}^{(u b)}$ is the upperbound of the maximum stopband error. Another parameter that is used interchangingly with $\Gamma_{s b}^{(u b)}$ is the lower-bound of the stopband attenuation and is defined as

$$
\Gamma_{a}^{(l b)}=-20 \log \Gamma_{s b}^{(u b)}
$$

For the circular model, the problem in (21) can be formulated as a convex optimization problem by introducing an auxiliary variable $t$ and using (19) and (20) for the stopband and 
passband errors, respectively:

minimize

$$
\begin{aligned}
& t \\
& \left|\sum_{n=0}^{N-1} \mathbf{b}_{n}\left(\omega_{i}, \theta_{p}\right) \mathbf{x}_{n}-B_{d}\left(\omega_{i}, \theta_{p}\right)\right|+ \\
& \sum_{n=0}^{N-1}\left|\mathbf{f}_{n}\left(\omega_{i}, \theta_{p}\right)^{T} \mathbf{x}_{n}\right| \leq t, \forall\left\{\begin{array}{l}
\theta_{p} \in \Theta_{p}, \\
\omega_{i} \in \Omega
\end{array}\right. \\
& \left|\sum_{n=0}^{N-1} \mathbf{b}_{n}\left(\omega_{i}, \theta_{s}\right) \mathbf{x}_{n}\right|+ \\
& \sum_{n=0}^{N-1}\left|\mathbf{f}_{n}\left(\omega_{i}, \theta_{s}\right)^{T} \mathbf{x}_{n}\right| \leq \Gamma_{s b}^{(u b)}, \forall\left\{\begin{array}{l}
\theta_{s} \in \Theta_{s} \\
\omega_{i} \in \Omega
\end{array}\right.
\end{aligned}
$$

where

$$
\begin{aligned}
\mathbf{b}_{n}(\omega, \theta) & =\mathbf{u}^{T} \mathbf{q}(\omega, \theta) \hat{\mathbf{g}}_{n}(\omega, \theta)^{T} \\
\mathbf{f}_{n}(\omega, \theta) & =\left\|\mathbf{P}(\omega, \theta)^{T} \mathbf{u}\right\|_{\infty} \hat{\mathbf{g}}_{n}(\omega, \theta)
\end{aligned}
$$

$B_{d}\left(\omega_{i}, \theta_{p}\right)$ is the desired response in the passband, and $\mathbf{x}_{0}, \ldots, \mathbf{x}_{N-1}$, and $t$ are the optimization variables. Note that if the desired response in the passband is linear phase with a prescribed group-delay of $\tau$, we have

$$
B_{d}\left(\omega_{i}, \theta_{p}\right)=e^{-j \omega_{i} \tau}
$$

Furthermore, if $\mathbf{x}_{0}^{(o p t)}, \ldots, \mathbf{x}_{N-1}^{(o p t)}$ are the optimal values of the problem in (23), the upper bound of the maximum passband error, derived from the microphone error-variation model, for the corresponding beamformer is given by

$$
\begin{aligned}
& \Gamma_{p b}^{(u b)}=\max _{\omega_{i}, \theta_{p}}\left\{\left|\sum_{n=0}^{N-1} \mathbf{b}_{n}\left(\omega_{i}, \theta_{p}\right) \mathbf{x}_{n}^{(o p t)}-B_{d}\left(\omega_{i}, \theta_{p}\right)\right|\right. \\
& \left.+\sum_{n=0}^{N-1}\left|\mathbf{f}_{n}\left(\omega_{i}, \theta_{p}\right)^{T} \mathbf{x}_{n}^{(o p t)}\right|\right\}
\end{aligned}
$$

\section{B. Two-phase Design Method}

In this design method, we seek to maximize the WNG, $G_{\omega}\left(\mathbf{x}, \omega_{i}\right)$, while using the maximum passband and stopband errors derived using the proposed method in Section III-A, as upper-bound constraints for the minimax problem. That is,

maximize $\quad \min _{\omega_{i}} G_{\omega}\left(\mathbf{x}, \omega_{i}\right)$

subject to: $\quad \max _{\omega_{i}, \theta_{p}}\left|B\left(\mathbf{x}, \omega_{i}, \theta_{p}\right)-B_{d}\left(\omega_{i}, \theta_{p}\right)\right| \leq \Gamma_{p b}^{(p h 1)}$ $\max _{\omega_{i}, \theta_{s}}\left|B\left(\mathbf{x}, \omega_{i}, \theta_{s}\right)\right| \leq \Gamma_{s b}^{(p h 1)}$

where

$$
\begin{aligned}
\Gamma_{p b}^{(p h 1)} & =\max _{\omega_{i}, \theta_{p}}\left|B\left(\mathbf{x}_{o p t 1}, \omega_{i}, \theta_{p}\right)-B_{d}\left(\omega_{i}, \theta_{p}\right)\right| \\
\Gamma_{s b}^{(p h 1)} & =\max _{\omega_{i}, \theta_{s}}\left|B\left(\mathbf{x}_{o p t 1}, \omega_{i}, \theta_{s}\right)\right|
\end{aligned}
$$

and $\mathbf{x}_{o p t 1}$ is the resulting beamformer solution of the proposed design method in (23).

The problem in (28) is, however, non-convex due to the non-convex cost function. To maximize the WNG as a convex optimization problem, we use the knowledge that the numerator of the WNG in (8) is approximately unity and therefore seek to minimize only the denominator of (8), which is a convex expression. Consequently, using an auxiliary variable $t$ the problem in (28) can be formulated as a convex optimization problem given by

$$
\begin{aligned}
\operatorname{minimize} & t \\
\text { subject to: } & \max _{\omega_{i}}\left\|\mathbf{A}\left(\omega_{i}\right) \mathbf{x}\right\|_{2} \leq t \\
& \max _{\omega_{i}, \theta_{p}}\left|\mathbf{g}\left(\omega_{i}, \theta_{p}\right) \mathbf{x}-B_{d}\left(\omega_{i}, \theta_{p}\right)\right| \leq \Gamma_{p b}^{(p h 1)} \\
& \max _{\omega_{i}, \theta_{s}}\left|\mathbf{g}\left(\omega_{i}, \theta_{s}\right) \mathbf{x}\right| \leq \Gamma_{s b}^{(p h 1)}
\end{aligned}
$$

where $t \in \mathbf{R}^{1}$ and $\mathbf{x} \in \mathbf{R}^{N L}$ are optimization variables. The procedure for the two-phase method can be summarized as follows:

Phase 1: Solve the optimization problem in (23). From the resulting beamformer solution, compute the passband and stopband error upper-bounds, given by (29) and (30), respectively.

Phase 2: Using the upper-bounds obtained in Phase 1, solve the optimization problem in (31) to obtain the beamformer.

Since the two-phase method requires two convex optimization problems to be solved it can therefore be considered to be twice the complexity of the proposed design method.

\section{Special case of symmetric FIR filters with symmetric mi- crophone array}

If the beampattern is symmetric about $\theta=\pi / 2$ in magnitude and phase, and the position of the array sensors are symmetric with respect to the array center such that

$$
d_{N-n-1}=-d_{n}
$$

then the filters of the beamformer will satisfy the symmetry condition [15]

$$
x_{n, l}=x_{N-n-1, l}
$$

Conversely, if the positions of the array sensors are symmetric with respect to the array center and condition (33) is satisfied, then the beampattern is always symmetric. Since (33) is an affine condition, it can therefore be incorporated in the convex optimization problem in (23) as an additional constraint. Note that the inclusion of the constraint in (33) eliminates possible solutions that do not exactly satisfy the symmetry conditions. In other words, it ensures that the beampattern is always symmetrical.

If the desired group delay is set to

$$
\tau_{h l f}=(L-1) f_{s}^{-1} / 2
$$

then the beamformer is guaranteed to be perfectly linear phase if (32) and the condition

$$
x_{n, l}=x_{N-n-1, L-l-1}
$$

are satisfied, regardless of whether or not the beampattern is symmetric [15]. Note that incorporating the conditions (32) and (35) in the optimization problem when the desired groupdelay, $\tau_{d}$, is set to $\tau_{h l f}$ ensures that the beamformer is perfectly linear phase only when there are no microphone errors; if microphone errors are present, the beamformer will cease to have perfect linear phase. 


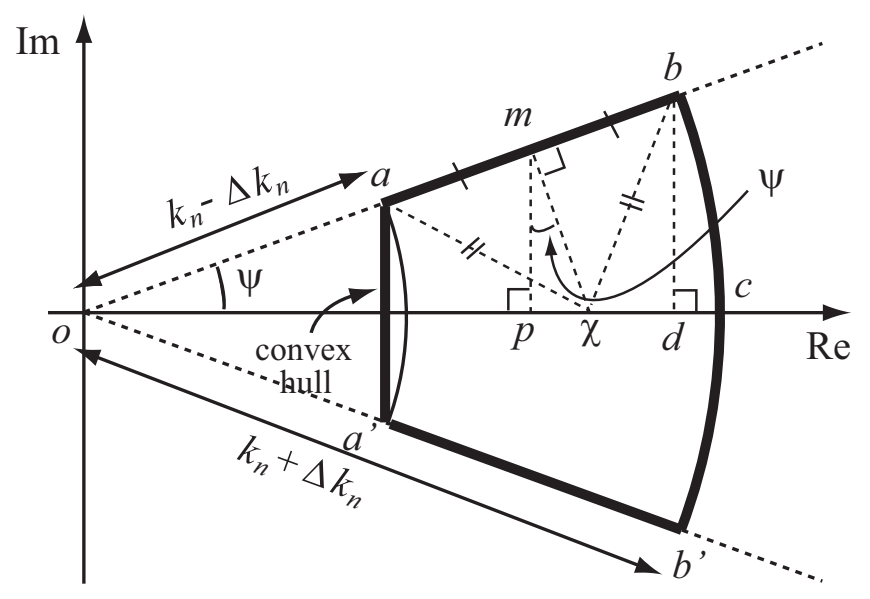

Fig. 3. Plot of the convex hull of $\kappa_{n}$ after rotation by $\alpha_{n}$.

\section{Computing the Parameters of the Microphone- ERROR-VARIATION MODEL}

In this section, we describe an efficient methods to compute the parameters of the circular model. As mentioned in Section III, the errors due the gain, phase, and position of the $n$th microphone manifest as a variation of $\kappa_{n}(\omega, \theta)$ that corresponds to a sector of an annulus in the complex plane. Since an annulus is clearly a non-convex set, it cannot be directly incorporated in a convex optimization problem. Therefore, to make it convex we form a convex hull of the annulus and model the hull using a minimum-area enclosing circle. Since the enclosing circle is required to be calculated for every sample of frequency and angle ${ }^{1}$, the computation should require low computational effort.

To simplify the computation of the minimum-area enclosing circle, we first rotate the convex hull, by say $\alpha_{n}$ radians, so that it is symmetric about the $\mathrm{x}$-axis (or real-axis) as shown in Fig. 3. Then we find the minimum-area enclosing circle for the hull, and after that rotate the circle back to the original position of the hull by $-\alpha_{n}$. Parameter $\alpha_{n}$ is obtained by taking the midpoint of the variation of the argument of $\kappa_{n}(\omega, \theta)$ in (12), which turns out to be

$$
\alpha_{n}=-\eta_{n}
$$

as evident from Fig. 2. From Fig. 3, the $x$ and $y$ coordinates for $a, a^{\prime}, b, b^{\prime}$, and $c$ are given by

$$
\begin{aligned}
x_{a} & =\left(k_{n}-\Delta k_{n}\right) \cos \psi \\
y_{a} & =\left(k_{n}-\Delta k_{n}\right) \sin \psi \\
x_{a^{\prime}} & =x_{a} \\
y_{a^{\prime}} & =-y_{a} \\
x_{b} & =\left(k_{n}+\Delta k_{n}\right) \cos \psi \\
y_{b} & =\left(k_{n}+\Delta k_{n}\right) \sin \psi
\end{aligned}
$$

\footnotetext{
${ }^{1}$ If there are no position errors, the model needs to be calculated only once, since it is identical for all sample points.
}

$$
\begin{aligned}
x_{b^{\prime}} & =x_{b} \\
y_{b^{\prime}} & =-y_{b} \\
x_{c} & =k_{n}+\Delta k_{n} \\
y_{c} & =0 \\
x_{d} & =\left(k_{n}+\Delta k_{n}\right) \cos \psi \\
y_{d} & =0
\end{aligned}
$$

where

$$
\psi=\Delta \eta_{n}+\left|\frac{\omega \Delta \hat{d}_{n} \cos \theta}{c}\right|
$$

In our method, we assume that the angular measure of the arc $b c b^{\prime}$ in Fig. 3 is less than 180 degrees, or $\psi<\pi / 2$. From our numerous experiments, we find that this assumption is reasonable since a beamformer that does not satisfy the condition will have very large errors that will make it practically ineffective. Therefore from Fig. 3, it is apparent that

$$
\operatorname{dist}(b, d)>\operatorname{dist}(c, d), \text { if } \psi<\pi / 2
$$

where $\operatorname{dist}\left(p_{1}, p_{2}\right)$ gives the distance between points $p_{1}$ and $p_{2}$. Furthermore, if point $k$ lies anywhere between $o$ and $c$ in line $o c$ then from Appendix B of [26] we have

$$
\operatorname{dist}(b, k)>\operatorname{dist}(c, k), \text { if } \psi<\pi / 2
$$

Therefore, if $\operatorname{dist}(b, d)>\operatorname{dist}(a, d)$ and knowing that (39) and (40) are true, it is clear that point $d$ is the center of the minimum-area circle, $\chi$, with coordinates and radius that are given by

$$
\begin{aligned}
& x_{\chi}=x_{b} \\
& y_{\chi}=0 \\
& r_{\chi}=y_{b}
\end{aligned}
$$

However, if $\operatorname{dist}(b, d)<\operatorname{dist}(a, d)$, then the center $\chi$ of the minimum-area circle will lie between $o$ and $c$ in line $o c$ such that $\operatorname{dist}(b, \chi)=\operatorname{dist}(a, \chi)$. As shown in Appendix $\mathrm{C}$ of [26], the coordinates and radius of the minimum-area circle are given by

$$
\begin{aligned}
& x_{\chi}=k_{n} \cos \psi+k_{n} \sin \psi \tan \psi \\
& y_{\chi}=0 \\
& r_{\chi}=\sqrt{\frac{k_{n}^{2}-k_{n}^{2} \cos ^{2} \psi+\Delta k_{n}^{2} \cos ^{2} \psi}{\cos ^{2} \psi}}
\end{aligned}
$$

In terms of the microphone error parameters, $\operatorname{dist}(b, d)$ and $\operatorname{dist}(a, d)$ can be expressed as

$$
\begin{aligned}
\operatorname{dist}(b, d)= & \left(k_{n}+\Delta k_{n}\right) \sin \psi \\
\operatorname{dist}(a, d)= & \left(3 \Delta k_{n}^{2} \cos ^{2} \psi+k_{n}^{2} \sin ^{2} \psi\right. \\
& \left.-2 k_{n} \Delta k_{n} \sin ^{2} \psi+\Delta k_{n}^{2}\right)^{0.5}
\end{aligned}
$$

and the condition $\operatorname{dist}(b, d)>\operatorname{dist}(a, d)$ is equivalent to the condition

$$
\Delta k_{n}^{2} \cos ^{2} \psi-\Delta k_{n} k_{n} \sin ^{2} \psi<0
$$

Consequently, the computation of the co-ordinates and radius of the minimum-area circle that encloses the convex hull $a b c b^{\prime} a^{\prime}$ can be carried out using the following steps: 
Step 1: Verify the condition in (49).

Step 2: If the condition in Step 1 is true, the co-ordinates and radius of the minimum area circle are given by eqns (41)(43), respectively.

Step 3: If the condition in Step 1 is false, the co-ordinates and radius of the minimum area circle are given by eqns (44)(46), respectively.

The resulting circle should then be rotated back by $-\alpha_{n}$ to cancel the initial pre-rotation by $\alpha_{n}$. The final rotated circular model in terms of the circular parameters $\mathbf{P}_{n}(\omega, \theta)$ and $\mathbf{q}_{n}(\omega, \theta)$ are given by

$$
\begin{aligned}
\mathbf{P}_{n}(\omega, \theta) & =\boldsymbol{\Phi}\left(-\alpha_{n}\right) \mathbf{P}^{\prime}(\omega, \theta) \\
\mathbf{q}_{n}(\omega, \theta) & =\boldsymbol{\Phi}\left(-\alpha_{n}\right) \mathbf{q}^{\prime}(\omega, \theta)
\end{aligned}
$$

where

$$
\begin{aligned}
\mathbf{P}^{\prime}(\omega, \theta) & =\left[\begin{array}{cc}
r_{\chi} & 0 \\
0 & r_{\chi}
\end{array}\right] \\
\mathbf{q}^{\prime}(\omega, \theta) & =\left[\begin{array}{ll}
x_{\chi} & y_{\chi}
\end{array}\right]^{T}
\end{aligned}
$$

and

$$
\boldsymbol{\Phi}(x)=\left[\begin{array}{cc}
\cos x & -\sin x \\
\sin x & \cos x
\end{array}\right]
$$

\section{EXPERIMENTAL RESUlTS}

In this section, we provide comparative experimental results between the proposed method, the two-phase method, and a modified version of the method in [19]. Though in most of the comparisons we set the group delay of the beamformer to $(L-1) f_{s}^{-1} / 2$, we also included some examples where the group delay is set to 0 . Consequently, we have two design variants for the proposed method and the two-phase method:

Design Pr-A: This design corresponds to the proposed beamformer design method that is obtained by setting the prescribed group-delay to $(L-1) f_{s}^{-1} / 2$ and solving the convex optimization problem in (23) while at the same time incorporating the linear-phase constraint in (35) and assuming that the condition in (32) is satisfied. For the symmetric case where $\theta_{d}=\pi / 2$, we also include the affine constraint in (33).

Design Pr-B: This design corresponds to the proposed beamformer design method that is obtained by setting the prescribed group-delay to 0 and solving the convex optimization problem in (23). For the symmetric case where $\theta_{d}=\pi / 2$, we solve the optimization problem by including the additional affine constraint in (33).

Design Tp-A: This design corresponds to the two-phase beamformer design method that is obtained by setting the prescribed group-delay to $(L-1) f_{s}^{-1} / 2$ and solving the convex optimization problem in (31) while at the same time incorporating the linear-phase constraint in (35) and assuming that the condition in (32) is satisfied. For the symmetric case where $\theta_{d}=\pi / 2$, we also include the affine constraint in (33).

Design Tp-B: This design corresponds to the two-phase beamformer design method that is obtained by setting the prescribed group-delay to 0 and solving the convex optimization problem in (31). For the symmetric case where $\theta_{d}=\pi / 2$, we solve the optimization problem by including the additional affine constraint in (33).
To ensure a fair comparison with the proposed method, we modify the method in [19] by moving the source to infinity so that the beamformer becomes far-field and formulate the optimization problem by minimizing the worst-case passband error under the constraint that the worst-case stopband error is above a prescribed level, as in (21). With these modifications, the modified method in [19] is given by

$$
\begin{aligned}
& \text { minimize } t \\
& \text { subject to: }\left|\mathbf{g}\left(\omega_{i}, \theta_{p}\right)^{T} \mathbf{x}-B_{d}\left(\omega_{i}, \theta_{p}\right)\right| \\
&+\epsilon\|\mathbf{x}\| \leq t \forall\left\{\begin{array}{l}
\theta_{p} \in \Theta_{p}, \\
\omega_{i} \in \Omega
\end{array}\right. \\
& \\
&\left|\mathbf{g}\left(\omega_{i}, \theta_{s}\right)^{T} \mathbf{x}\right| \\
&+\epsilon\|\mathbf{x}\| \leq \Gamma_{s b}^{(u b)}, \forall\left\{\begin{array}{l}
\theta_{s} \in \Theta_{s}, \\
\omega_{i} \in \Omega
\end{array}\right.
\end{aligned}
$$

where

$$
\begin{aligned}
\epsilon & = \begin{cases}\mathcal{F}(\Delta k, \Delta \eta) & \text { for gain and phase errors } \\
\mathcal{G}(\Delta d) & \text { for position errors }\end{cases} \\
\mathcal{F}(\Delta k, \Delta \eta) & =\sqrt{N L[(1+\Delta k)(1+\Delta k-2 \cos \Delta \eta)+1]} \\
\mathcal{G}(\Delta d) & =\sqrt{N L+\frac{N L}{(1-\Delta d)^{2}}-\frac{2 N L \cos \left(\frac{2 \pi \Delta d}{\lambda_{\text {min }}}\right)}{1-\Delta d}}
\end{aligned}
$$

$\lambda_{\min }$ is the minimum wavelength in the frequency band and $\Delta k, \Delta \eta$, and $\Delta d$ are the maximum absolute variation of the gain, phase, and position errors, respectively, from their ideal values for all the sensors. The corresponding upper bound of maximum passband error for the beamformer derived from (55) is given by

$$
\Gamma_{p b}^{(u b)}=\max _{\omega_{i}, \theta_{p}}\left\{\left|\mathbf{g}\left(\omega_{i}, \theta_{p}\right)^{T} \mathbf{x}^{(o p t)}-B_{d}\left(\omega_{i}, \theta_{p}\right)\right|+\epsilon\left\|\mathbf{x}^{(o p t)}\right\|\right\}
$$

Consequently, the modified method in [19] can be classified into two variants:

Design $C p$-A: This design corresponds to the modified competing method in [19] that is obtained by setting the prescribed group-delay to $(L-1) f_{s}^{-1} / 2$ and solving the convex optimization problem in (55) while at the same time incorporating the linear-phase constraint in (35) and assuming that the condition in (32) is satisfied. For the symmetric case where $\theta_{d}=\pi / 2$, we also include the affine constraint in (33).

Design $C p-B$ : This design corresponds to the modified competing method in [19] that is obtained by setting the prescribed group-delay to 0 and solving the convex optimization problem in (55). For the symmetric case where $\theta_{d}=\pi / 2$, we solve the optimization problem by including the additional affine constraint in (33).

In the experiments, we consider design examples where the beampatterns are symmetric and nonsymmetric about $\theta=\pi / 2$. For the symmetric case, the desired steering angle $\theta_{d}$, which is used in (8), is set to $\pi / 2$ and for the nonsymmetric case to $2 \pi / 3$. The speed of sound, $c$, is assumed to be $340 \mathrm{~m} / \mathrm{s}$ while the sampling frequency, $f_{s}$, is assumed to be $8 \mathrm{kHz}$. The parameters are evaluated by uniformly sampling the frequency and angular bands and setting the number of sampling points 
$M$ and $K$ along the two dimensions to each have a value of 120.

The beamformer performance is evaluated using the following parameters:

Maximum passband error: The maximum passband error is defined as

$$
\delta_{p}=\max _{\omega \in \Omega, \theta \in \Theta_{p}}\left|B(\omega, \theta)-B_{d}(\omega, \theta)\right|
$$

where $B_{d}(\omega, \theta)$ is the desired beamformer response in the passband.

Maximum passband ripple: The parameter is defined as

$$
A_{p}=20 \log \frac{M_{\max }^{(p)}}{M_{\min }^{(p)}}
$$

where

$$
\begin{aligned}
& M_{\text {max }}^{(p)}=\max _{\omega \in \Omega, \theta \in \Theta_{p}} B(\omega, \theta) \\
& M_{\text {min }}^{(p)}=\min _{\omega \in \Omega, \theta \in \Theta_{p}} B(\omega, \theta)
\end{aligned}
$$

Minimum stopband attenuation: The minimum stopband attenuation is defined as the negative of the maximum stopband gain, given by

$$
A_{a}=-20 \log M_{\max }^{(a)}
$$

where

$$
M_{\max }^{(a)}=\max _{\omega \in \Omega, \theta \in \Theta_{s}} B(\omega, \theta)
$$

To evaluate the beamformer performance due to the microphone errors, we randomly vary the gain, phase, and position of each of the $n$ microphones about their ideal values by $\pm \Delta \kappa_{n}, \pm \Delta \eta_{n}$, and $\pm \Delta d_{n}$, respectively. Therefore, each trial is a random combination of the worst-case sensor variations about their ideal values. For each trial, we compute the beamformer performance parameters in (57), (58), and (59) and then compute their worst values among all the trials as

$$
\begin{aligned}
\delta_{p}^{(w c)} & =\max _{i=1, \ldots, \mathcal{T}} \delta_{p}^{(i)} \\
A_{p}^{(w c)} & =\max _{i=1, \ldots, \mathcal{T}} A_{p}^{(i)} \\
A_{a}^{(w c)} & =\min _{i=1, \ldots, \mathcal{T}} A_{a}^{(i)}
\end{aligned}
$$

where $\delta_{p}^{(i)}, A_{p}^{(i)}$, and $A_{a}^{(i)}$ are the corresponding performance values in the $i$ th trial. In our simulations, we set the total number of trials, $\mathcal{T}$, to 100 .

In Sections V-A to V-D, nine examples are provided to compare the performance of the proposed method with the competing methods. In Examples 1 and 2 we assume that the beamformers have gain/phase errors and position error, respectively, and compare designs $\mathrm{Pr}-\mathrm{A}$ with $\mathrm{Cp}$ - A. Then in Examples 3 and 4, we consider beamformers that have gain, phase, and position errors and compare designs Pr-A with TpA. In Examples 5, 6, and 7 we again consider beamformers

\begin{tabular}{|c|c|c|}
\hline Parameters & \multicolumn{2}{|c|}{ Common values } \\
\hline No. of elements of beamformer & \multicolumn{2}{|l|}{7} \\
\hline Inter-element spacing, (m) & \multicolumn{2}{|l|}{0.04} \\
\hline FIR filter length & \multicolumn{2}{|l|}{20} \\
\hline Passband region, $\Theta_{p},(\mathrm{deg})$ & \multicolumn{2}{|c|}{$\left[80^{\circ}-100^{\circ}\right]$} \\
\hline Stopband region, $\Theta_{s}$, (deg) & \multicolumn{2}{|c|}{$\left[0^{\circ}-60^{\circ}\right] \cup\left[120^{\circ}-180^{\circ}\right.$} \\
\hline Frequency band, $\Omega,(\mathrm{Hz})$ & \multicolumn{2}{|c|}{$[1500-3500]$} \\
\hline LB of SB atten., $\Gamma_{a}^{(l b)}(\mathrm{dB})$ & \multicolumn{2}{|l|}{$\begin{array}{l}6 \\
95\end{array}$} \\
\hline Passband group delay (samples) & \multicolumn{2}{|l|}{9.5} \\
\hline & $\begin{array}{l}\text { Example 1 } \\
\text { values }\end{array}$ & $\begin{array}{l}\text { Example } 2 \\
\text { values }\end{array}$ \\
\hline Mic. gain error, $\left(k_{n}, \Delta k_{n}\right)$ & $(1,0.05)$ & $(1,0)$ \\
\hline Mic. phase error, $\left(\eta_{n}, \Delta \eta_{n}\right)(\mathrm{deg})$ & $(0,5)$ & $(0,0)$ \\
\hline Mic. posn. error, $\left(\hat{d}_{n}, \Delta \hat{d}_{n}\right)(\mathrm{m})$ & $\left(d_{n}, 0\right)$ & $\left(d_{n}, 0.001\right)$ \\
\hline
\end{tabular}
that have gain, phase, and position errors and compare designs Pr-A with Tp-A; we also compare designs Pr-B with Tp$\mathrm{B}$ in Examples 5 and 6. In Examples 8 and 9, we assume beamformers that have gain error and phase error, respectively, and compare designs Pr-A with Cp-A.
TABLE I

DESIGN SPECIFICATIONS FOR BEAMFORMERS SYMMETRIC ABOUT $\theta=\pi / 2$ FOR EXAMPLES 1 AND 2

TABLE II

DESIGN RESULTS FOR EXAMPLE 1 FOR A SYMMETRIC BEAMFORMER WITH GAIN AND PHASE ERRORS.

\begin{tabular}{||l|c|c||}
\hline \hline Parameters & $\begin{array}{c}\text { Design } \\
\text { Pr-A }\end{array}$ & $\begin{array}{c}\text { Design } \\
\text { Cp-A }\end{array}$ \\
UB of max PB error $\Gamma_{p b}^{(u b)}$ & 0.207 & 0.673 \\
WC max PB error $\delta_{p}^{(w c)}$ & 0.144 & 0.444 \\
WC max PB ripple $A_{p}^{(w c)}, \mathrm{dB}$ & 1.87 & 2.92 \\
WC min SB atten. $A_{a}^{(w c)}, \mathrm{dB}$ & 7.04 & 11.23 \\
\hline
\end{tabular}

PB: passband; SB: stopband; WC: worst-case; UB: upper-bound

\section{A. Examples 1 and 2}

In this subsection, we consider the design of beamformers where the passband group delay is set to $(L-1) f_{s}^{-1} / 2$. We compare their performance by observing the design that results in the smallest worst-case maximum-passband-error $\delta_{p}^{(w c)}$ while ensuring that the worst-case minimum-stopbandattenuation $A_{a}^{(w c)}$ is above the prescribed level of $\Gamma_{a}^{(l b)}$; in addition, we also compare the maximum passband ripple $A_{p}^{(w c)}$. For the comparisons, we consider designs Pr-A and Cp-A.

Two beamformer design examples are chosen. In the first example, the beamformer is assumed to have gain and phase errors while in the second example it has position errors. The design specifications for the first and second examples are given in Table I. The comparison results for Examples 1 and 2 are summarized in Tables II and III, respectively, and their corresponding beamformer responses and WNGs are plotted in Figs. 4 and 5, respectively. From Tables II and III and Figs. 4 and 5, we observe that the proposed designs have much smaller

TABLE III

DESIGN RESULTS FOR EXAMPLE 2 FOR A SYMMETRIC BEAMFORMER WITH POSITION ERRORS.

\begin{tabular}{||l|c|c||}
\hline \hline Parameters & $\begin{array}{c}\text { Design } \\
\text { Pr-A }\end{array}$ & $\begin{array}{c}\text { Design } \\
\text { Cp-A }\end{array}$ \\
UB of max PB error $\Gamma_{p b}^{(u b)}$ & 0.044 & 0.449 \\
WC max PB error $\delta_{p}^{(w c)}$ & 0.0437 & 0.213 \\
WC max PB ripple $A_{p}^{(w c)}, \mathrm{dB}$ & 0.713 & 2.38 \\
WC min SB atten. $A_{a}^{(w c)}, \mathrm{dB}$ & 6.18 & 11.36 \\
\hline
\end{tabular}

PB: passband; SB: stopband; WC: worst-case; UB: upper-bound 

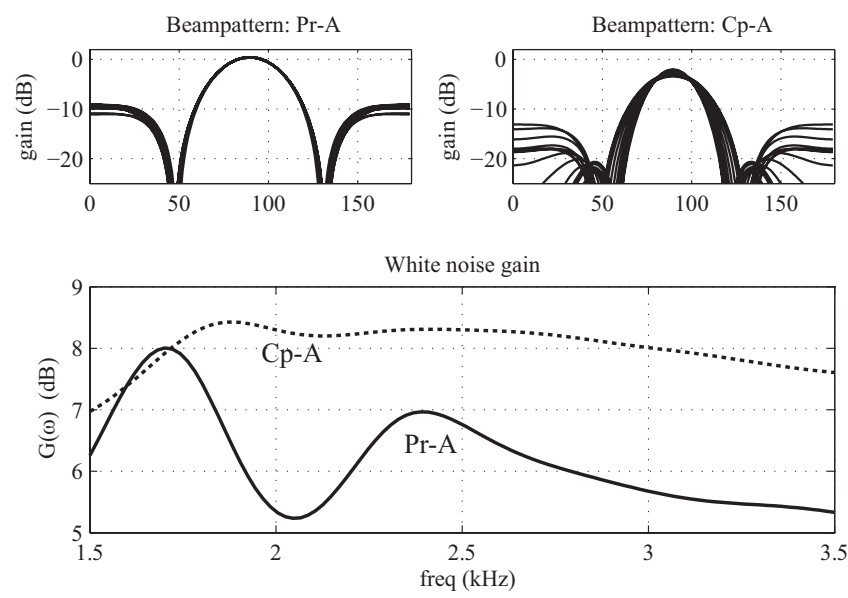

Fig. 4. Plots of the beamformer response and white noise gain for the various designs for Example 1 when there are no sensor errors. The beampattern plots are obtained by plotting the responses across 20 uniformly sampled frequencypoints in the frequency band.
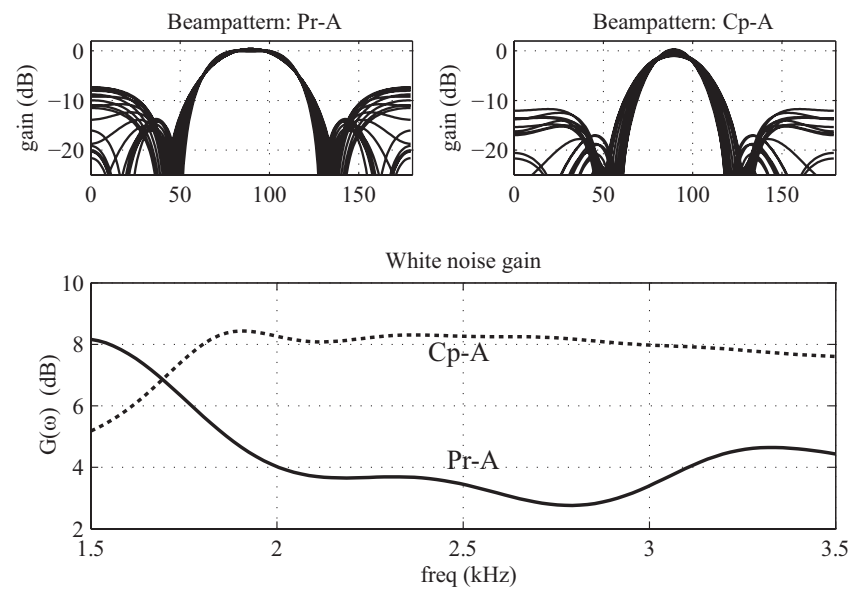

Fig. 5. Plots of the beamformer response and white noise gain for the various designs for Example 2 when there are no sensor errors. The beampattern plots are obtained by plotting the responses across 20 uniformly sampled frequencypoints in the frequency band.

worst-case passband error, passband ripple, and passband error upperbound than the competing designs; furthermore, the worst-case stopband attenuations of the proposed designs are much closer to the prescribed lower bound of $6 \mathrm{~dB}$ than the competing designs.

\section{B. Examples 3 and 4}

Here we consider the design of beamformers where the passband group delay is set to $(L-1) f_{s}^{-1} / 2$. In the first example, the beamformer is symmetric about $\theta=\pi / 2$ while in the second example it is non-symmetric. The design specifications for Examples 3 and 4 are given in Table IV. The comparison results for Examples 3 and 4 are summarized in Tables V and VI, respectively, and their corresponding beamformer response and WNG plots are provided in [26]. For Example 3, we observe from Table V that designs Pr-A and $\mathrm{Tp}$-A have comparable performance, with their worst-case stopband attenuation, $A_{a}^{(w c)}$, satisfying the $6 \mathrm{~dB}$ lower-bound
TABLE IV

DESIGN SPECIFICATIONS FOR BEAMFORMERS SYMMETRIC AND NONSYMMETRIC ABOUT $\theta=\pi / 2$ FOR EXAMPLES 3 AND 4 , RESPECTIVELY

\begin{tabular}{|c|c|c|}
\hline Parameters & \multicolumn{2}{|l|}{ Common values } \\
\hline No. of elements of beamformer & \multicolumn{2}{|l|}{7} \\
\hline Inter-element spacing, (m) & \multicolumn{2}{|l|}{0.04} \\
\hline FIR filter length & \multicolumn{2}{|l|}{20} \\
\hline Frequency band, $\Omega,(\mathrm{Hz})$ & \multirow{2}{*}{\multicolumn{2}{|c|}{$[1500-3500]$}} \\
\hline LB of SB atten., $\Gamma_{a}^{(l b)}(\mathrm{dB})$ & & \\
\hline Passband group delay (samples) & \multicolumn{2}{|l|}{9.5} \\
\hline Mic. gain error, $\left(k_{n}, \Delta k_{n}\right)$ & \multicolumn{2}{|l|}{$(1,0.05)$} \\
\hline Mic. phase error, $\left(\eta_{n}, \Delta \eta_{n}\right)(\mathrm{deg})$ & \multicolumn{2}{|l|}{$(0,5)$} \\
\hline Mic. posn. error, $\left(\hat{d}_{n}, \Delta \hat{d}_{n}\right)(\mathrm{m})$ & \multicolumn{2}{|l|}{$\left(d_{n}, 0.001\right)$} \\
\hline & $\begin{array}{l}\text { Example } 3 \\
\text { values }\end{array}$ & $\begin{array}{l}\text { Example } 4 \\
\text { values }\end{array}$ \\
\hline $\begin{array}{l}\text { Passband region, } \Theta_{p},(\mathrm{deg}) \\
\text { Stopband region, } \Theta_{s},(\mathrm{deg})\end{array}$ & $\begin{array}{l}{\left[80^{\circ}-100^{\circ}\right]} \\
{\left[0^{\circ}-60^{\circ}\right] \cup} \\
{\left[120^{\circ}-180^{\circ}\right]}\end{array}$ & $\begin{array}{l}{\left[110^{\circ}-130^{\circ}\right]} \\
{\left[0^{\circ}-90^{\circ}\right] \cup} \\
{\left[150^{\circ}-180^{\circ}\right]}\end{array}$ \\
\hline
\end{tabular}

SB: stopband; LB: lower-bound

TABLE V

DESIGN RESULTS FOR EXAMPLE 3 FOR A SYMMETRIC BEAMFORMER WITH GAIN, PHASE, AND POSITION ERRORS.

\begin{tabular}{||l|c|c||}
\hline \hline Parameters & $\begin{array}{c}\text { Design } \\
\text { Pr-A }\end{array}$ & $\begin{array}{c}\text { Design } \\
\text { Tp-A }\end{array}$ \\
\hline UB of max PB error $\Gamma_{p b}^{(u b)}$ & 0.223 & NA \\
WC max PB error $\delta_{p}^{(w c)}$ & 0.153 & 0.154 \\
WC max PB ripple $A_{p}^{(w c)}, \mathrm{dB}$ & 2.11 & 1.97 \\
WC min SB atten. $A_{a}^{(w c)}, \mathrm{dB}$ & 7.51 & 7.62 \\
\hline
\end{tabular}

PB: passband; SB: stopband; WC: worst-case; UB: upper-bound

requirement. We also observe that though design Pr-A has a better value of $\delta_{p}^{(w c)}$, design Tp-A has a better value of $A_{p}^{(w c)}$. For Example 4, we observe from Table VI that designs Pr-A and Tp-A have comparable performance with $A_{a}^{(w c)}$ satisfying the $6 \mathrm{~dB}$ lower-bound requirement for both the designs; as can be seen, design Pr-A has a better value of $A_{p}^{(w c)}$ while design Tp-A has a better value of $\delta_{p}^{(w c)}$. Furthermore, from Tables V and VI we observe that the beamformers derived using design Pr-A are certified with an upper-bound on the maximum passband error. This information can be quite useful to the designer when assessing the feasibility of a beamformer for a particular application.

\section{Examples 5, 6, and 7}

For Examples 5 and 6, we vary the design specification in Examples 3 and 4 by reducing the passband region and increasing the stopband attenuation. In addition, we also

TABLE VI

DESIGN RESULTS FOR EXAMPLE 4 FOR A NON-SYMMETRIC BEAMFORMER WITH GAIN, PHASE, AND POSITION ERRORS.

\begin{tabular}{||l|c|c||}
\hline \hline Parameters & $\begin{array}{c}\text { Design } \\
\text { Pr-A }\end{array}$ & $\begin{array}{c}\text { Design } \\
\text { Tp-A }\end{array}$ \\
\hline UB of max PB error $\Gamma_{p b}^{(u b)}$ & 0.377 & NA \\
WC max PB error $\delta_{p}^{(w c)}$ & 0.292 & 0.272 \\
WC max PB ripple $A_{p}^{(w c)}, \mathrm{dB}$ & 2.97 & 3.5 \\
WC min SB atten. $A_{a}^{(w c)}, \mathrm{dB}$ & 7.26 & 7.23 \\
\hline
\end{tabular}

PB: passband; SB: stopband; WC: worst-case; UB: upper-bound 
consider beamformers where the prescribed group delay is set to 0 . Consequently, we have four design variants for comparison, namely, designs Pr-A, Pr-B, Tp-A, and Tp-B. For Example 7, we vary the design specification in Example 3 by changing the frequency band to $[300-1500] \mathrm{Hz}$, increasing the distance between the sensors to $0.1 \mathrm{~m}$, and reducing the passband region; for the comparison we consider designs Pr$\mathrm{A}$ and Tp-A. The design specifications, results, and plots for the three examples are included in [26]. From the results and plots, we observe that though there are variations in the performance parameters between the designs, all four designs have comparable performances.

\section{Examples 8 and 9}

In Examples 8, the beamformers are assumed to have only gain errors, while in Example 9, only phase errors. For comparison, we consider design Pr-A with $\mathrm{Cp}$-A. The design specifications, results, and plots for both the examples are included in [26]. As in Examples 1 and 2, it is apparent from the results and plots that the proposed designs have much smaller worst-case passband errors, passband ripples, and passband error upperbounds than the competing designs. In addition, the worst-case stopband attenuations of the proposed designs are also much smaller and closer to the prescribed lower bound than the competing designs.

The comparisons in Examples 1-9 above have shown that the proposed method yields beamformers with much better performance than the modified method in [19] and comparable performance with the two-phase method. There are two main reasons why the proposed method performs better than the method in [19]: (i) The norm bound of the sensor imperfections in [19, eqn. (7)] correspond to a vector of length $N L$ where $N$ is the number of microphones and $L$ is the filter length. In our proposed method, the circular bound of the sensor imperfections in the complex plane corresponds to a norm bound of a vector of length 2 . In general, as the dimension of the bounded vector decreases, the bound becomes tighter. (ii) For the scenario where the beamformer has position errors, the method in [19] computes the bound at all frequencies by considering the minimum wavelength of the frequency band. In our proposed method, the bound at a given frequency is computed by considering the wavelength at that frequency thereby significantly improving the tightness of the bound.

From Examples 3-7, it has been observed that for the proposed method and the two-phase method, the worst-case passband and stopband error values obtained from 100 random trials are not far off from the corresponding worstcase passband and stopband error bounds computed from the model. It should be noted that the microphone error-variation model that has been proposed is a convex approximation of the microphone-error variations, and, therefore, the worstcase performance parameters predicted by the model will be slightly on the conservative side. Therefore, to compensate for this slightly conservative estimate, a good rule of thumb that we can suggest when designing beamformers using the proposed method is to reduce the lower-bound of the minimum stopband attenuation by a certain value typically between 0.5 and $1.5 \mathrm{~dB}$ from the actual lower-bound that is desired.

We would also like to point out that unlike the proposed method, the two-phase method provides no theoretical guarantees that the beamformer that is obtained will always satisfy the worst-case passband and stopband error bounds as it is derived by maximizing the WNG; however, in all our experiments we observe that the two-phase method always yields beamformers that give comparable performance to beamformers derived using the proposed method and, in addition, always satisfies the worst-case error bounds of the proposed method.

The optimization problems in the examples were solved on a computer running an Intel Core i7-640LM processor using the SeDuMi optimization toolbox for MATLAB [27]. For the proposed method, the optimization problem takes anywhere between 10 to 40 minutes to compute.

\section{CONCLUSIONS}

A new method for the design of minimax broadband beamformers that are robust to microphone gain, phase, and position errors has been proposed. In the method, the maximum variation in the microphone errors are used to formulate a convex optimization problem where the worst-case passband error is minimized under the constraint that the worst-case stopband error is below a prescribed level. To include the microphone imperfections in the optimization problem, a suitable model that incorporates the variations due to the microphone errors was developed, including an efficient method to compute the model. An important advantage of the proposed method is the availability of corresponding worst-case passband- and stopband-error bounds for the beamformer that has been designed; a second advantage is that it does not require the distribution of microphone errors. We then described a twophase method where the proposed method is used in the first phase to derive the passband and stopband error constraints for solving an optimization problem in the second phase where the white noise gain (WNG) of the beamformer is maximized. In our experiments, we compared beamformers designed using the proposed method, the two-phase method and a modified version of a competing method. Experimental results showed that beamformers designed using the proposed method have much better performance than those of the modified competing method and comparable performance with those of the twophase method; however, unlike two-phase method, the proposed method provides the additional guarantee that the errors will always lie within the worst-case error bounds.

\section{ACKNOWLEDGMENT}

I thank Dr. N. Ono, who coordinated the review, and the anonymous reviewers for their contributions to improving the quality of this paper.

\section{REFERENCES}

[1] M. Brandstein and D. Ward, Microphone Arrays: Signal Processing Techniques and Applications, New York: Springer 2001.

[2] E. Hansler and G. Schmidt, Acoustic echo and noise control - A practical approach, Wiley-Interscience 2004 
[3] D. B.Ward, Z. Ding, and R. A. Kennedy, "Broadband DOA estimation using frequency invariant beamforming," IEEE Trans. Signal Process., vol. 46, no. 5, pp. 1463-1469, May 1998.

[4] D. B. Ward, R. A. Kennedy, and R. C. Williamson, "FIR filter design for frequency-invariant beamformers," IEEE Signal Process. Lett., vol. 3, pp. 69-71, Mar. 1996.

[5] S. E. Nordholm, V. Rehbock, K. L. Teo, and S. Nordebo, "Chebyshev optimization for the design of broadband beamformers in the near field," IEEE Trans. Circuits Syst. II, Analog Digit. Signal Process., vol. 45, no. 1, pp. 141-143, Jan. 1998.

[6] B. K. Lau, Y. H. Leung, K. L. Teo, and V. Sreeram, "Minimax filters for microhpone arrays," IEEE Trans. Circuits Syst. II, Analog Digit. Signal Process., vol. 46, no. 12, pp. 1522-1525, Dec. 1999.

[7] K. F. C. Yiu, X. Yang, S. Nordholm, and K. L. Teo, "Near-field broadband beamformer design via multidimensional semi-infinite linear programming techniques," IEEE Trans. Speech Audio Process., vol. 11, no. 6 , pp. $725-732$, Nov. 2003.

[8] S. Doclo and M. Moonen, "Design of far-field and near-field broadband beamformers using eigenfilters," Signal Process., vol. 83, pp. 2641-2673, Dec. 2003.

[9] H. Cox, R. Zeskind, and T. Kooij, "Practical supergain," IEEE Trans. Acoust. Speech Signal Process., vol. ASSP-34, no. 3, pp. 393-398, Jun. 1986.

[10] J. M. Kates, "Superdirective arrays for hearing aids," J. Acoust. Soc. Amer, vol. 94, pp. 1930-1933, Oct. 1993.

[11] J. Bitzer and K. U. Simmer, "Superdirective microphone arrays," in Microphone Arrays: Signal Processing Techniques and Applications, M. S. Brandstein and D. B. Ward, Eds. New York: Springer-Verlag, May 2001, ch. 2, pp. 19-38.

[12] D. J. Shpak and A. Antoniou, "A flexible optimization method for the pattern synthesis of equispaced linear arrays with equiphase excitation," IEEE Trans. Antennas Propagat., vol. 40, pp. 1113-1120, Oct. 1992.

[13] D. J. Shpak, "A method for the optimal pattern synthesis of linear arrays with prescribed nulls," IEEE Trans. Antennas Propagat., vol. 44, pp. 286-294, Mar. 1996.

[14] Y. Zhao and W. Liu "Robust fixed frequency invariant beamformer design subject to norm-bounded errors," IEEE Signal Process. Lett., vol. 20, no. 2, pp. 169-172 2013.

[15] R. C. Nongpiur and D. J. Shpak, " $L$-infinity norm design of linearphase robust broadband beamformers using constrained optimization," IEEE Trans. Signal Process., vol. 61, no. 23, pp. 6034-6046, Dec. 2013.

[16] E. Mabande, A. Schad, and W. Kellermann, "Design of robust superdirective beamformer as a convex optimization problem," in Proc. Int. Conf. Acoust., Speech, Signal Process., Taipei, Taiwan, Apr. 2009.

[17] E. Mabande, A. Schad, and W. Kellermann, "A time-domain implementation of data-independent robust broadband beamformers with low filter order," in Proc. Joint Workshop on Hands-free Speech Communication and Microphone Arrays, Edinburgh, May 2011.

[18] E. Mabande, M. Buerger, and W. Kellermann, "Design of robust polynomial beamformers for symmetric arrays," in Proc. Int. Conf. Acoust., Speech, Signal Process., Vancouver, Canada, Mar. 2012.

[19] H. Chen, W. Ser, and Z. L. Yu, "Optimal design of nearfield wideband beamformers robust against errors in microphone array characteristics," IEEE Trans. Circuit Syst., vol. 54, pp. 1950-1959, Sep. 2007.

[20] H. Chen and W. Ser,, "Design of robust broadband beamformers with passband shaping characteristics using Tikhonov regularization," IEEE Trans. Audio, Speech, Lang. Process., vol. 17, no. 4, pp. 665-681, May 2009.

[21] M. Crocco and A. Trucco, "A computationally efficient procedure for the design of robust broadband beamformers," IEEE Trans. Signal Process., vol. 58, no. 10 , pp. 5420-5424, Oct. 2010.

[22] M. Crocco and A. Trucco, "Design of robust superdirective arrays with a tunable tradeoff between directivity and frequency-invariance," IEEE Trans. Signal Process., vol. 59, no. 5, pp. 2169-2181, May 2011.

[23] M. Crocco and A. Trucco, "Stochastic and analytic optimization of sparse aperiodic arrays and broadband beamformers with robust superdirective patterns," IEEE Trans. Audio, Speech, Lang. Process., vol. 20, no. 9, pp. 2433-2447, Nov. 2012.

[24] H. Chen, W. Ser, and J. Zhou, "Robust nearfield wideband beamformer design using worst case mean performance optimization with passband response variance constraint," IEEE Trans. Audio, Speech, Lang. Process., vol. 20, no. 5, pp. 1565-1572, Jul. 2012.

[25] S. Doclo and M. Moonen, "Design of broadband beamformers robust against gain and phase errors in the microphone array characteristics," IEEE Trans. Signal Process., vol. 51, no. 10, pp. 2511-2526, Oct. 2003.

[26] [Online]. Available: https://googledrive.com/host/0B6zSDcu_8P_ EUjFCUVIWSFF2X3c/addDocTaslp2014_1.pdf

[27] J. F. Sturm, "Using SeDuMi1.02, a MATLAB toolbox for optimization over symmetric cones," Optim. Methods Softw., vol. 11-12, pp. 625-653,
1999.

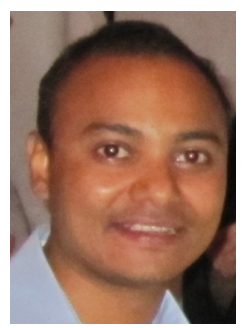

Rajeev C. Nongpiur (S'01, AM'05, M'12) received the B.Tech. degree in Electronics and Communications Engineering from the Indian Institute of Technology, Kharagpur, India, in 1998 and the Ph.D. degree from the University of Victoria, British Columbia, Canada, in 2005. From 1998 to 2000 he worked as Systems Engineer at Wipro Technologies, from 2004 to 2008 as Research Scientist at QNX Software Systems, and from 2008 to 2010 as Senior DSP Engineer at Unication Co., Ltd., Vancouver, Canada. He is currently serving as Research Associate in the Department of Electrical and Computer Engineering, University of Victoria, British Columbia, Canada. His research interests are in the areas of signal processing for digital communications, multimedia, and biomedical applications.

Dr. Nongpiur is a member of the IEEE Circuits and Systems and Signal Processing Societies. 